\title{
Valoración económica de las medidas destinadas a la recuperación ambiental de una masa de agua muy modificada. El caso del acuífero Bajo-Almanzora (Almería)
}

\author{
Almudena Gómez-Ramos ${ }^{\mathrm{a}}$ y Sofía Olmedilla Pérez
}

\begin{abstract}
RESUMEN: El nuevo proceso de planificación promovido por la Directiva Marco del Agua refuerza la necesidad de alcanzar los objetivos ambientales de las masas de agua mediante un programa de medidas. Este trabajo traslada este principio a la recuperación del acuífero Bajo Almanzora (Almería), masa muy modificada debido a la presión proveniente de la contaminación por nitratos. El trabajo aplica el análisis coste-eficacia a las medidas valorando los costes directos e indirectos en la comarca y en la provincia. Los resultados muestran la importancia de implementar medidas que promuevan la eficiencia en la fertilización una vez que se han aplicado las medidas complementarias.
\end{abstract}

PALABRAS CLAVES: Aguas subterráneas, análisis coste-eficacia, contaminación nitratos, Directiva Marco del Agua, objetivos ambientales.

Clasificación JEL: Q25.

DOI: $10.7201 /$ earn.2012.02.01.

Economic assesment of measures for environmental recovery of excessively modified water masses. The case of Bajo Almanzora aquifer (Almeria)

ABSTRACT: The new water planning process promoted by the Water Frame Directive strengthens the need to achieve environmental objectives for each water mass through an assessment of a program of measures. The current study applies this WFD's principle to the environmental recovery of an aquifer too deteriorated due to the pressure caused by nitrate pollution. This work applies cost-efficiency analysis to value direct and indirect costs caused by some measures for both the area and the province. Results show the importance of measures devoted to promote fertilization efficiency once complementary measures are implemented.

KEYWORDS: Groundwater, cost-efficiency analysis, nitrates pollution, Water Framework Directive, environmental objectives.

JEL classification: Q25.

DOI: $10.7201 /$ earn.2012.02.01.

Departamento de Ingenierías Agrarias. Área de Economía, Sociología y Política Agraria. Universidad de Valladolid.

b Subdirección General de Análisis, Prospectiva y Coordinación. Subsecretaría. MAGRAMA.

Dirigir correspondencia a: Almudena Gómez-Ramos. E-mail: almgomez@iaf.uva.es.

Recibido en mayo de 2011. Aceptado en mayo de 2012. 


\section{Introducción}

La revisión de la caracterización del uso del agua en las diferentes demarcaciones españolas pone de manifiesto el importante deterioro ambiental de algunas masas de agua, proveniente en la mayor parte de los casos de un uso intensivo de los recursos. Esta situación es especialmente patente en las masas de agua subterráneas sobreexplotadas de las cuencas mediterráneas, debido al crecimiento del riego intensivo en zonas áridas y semiáridas en los últimos cuarenta años (Sauquillo et al., 2009; Garrido et al., 2006; Almasri, 2007). Este hecho, que ha llegado a ser denominada "revolución silenciosa" (Llamas y Martínez-Santos, 2005), ha sido llevada a cabo por pequeños y medianos agricultores en un contexto de práctica ausencia de control y planificación por parte de las autoridades de cuenca encargadas de su gestión. Sin embargo y de forma simultánea, este modelo de explotación se ha visto acompañado por la generación de beneficios sociales y económicos en el área de influencia (Sánchez-Picón et al., 2011).

En este contexto surge la Directiva Marco del Agua (Directiva 2000/60 CE, DMA en adelante) que se aprueba con el objeto de conseguir el buen estado de las masas de agua en la UE en el año 2015. La Directiva establece que es preciso prevenir el deterioro adicional de ecosistemas acuáticos, promover el uso sostenible del agua, incrementar la protección del medio acuático y reducir de forma progresiva la contaminación de las aguas subterráneas. De este modo, los objetivos ambientales pasan ahora a ser prioritarios en relación a los objetivos de atención de demandas que incluyen las solicitudes de volúmenes de agua por parte de actividades socio-económicas (Maestu y Domingo, 2008).

El cumplimiento de los objetivos de la DMA exige a los Estados Miembro transferir estos principios a la gestión del agua a través de su incorporación al diseño de los Planes de las demarcaciones hidrográficas. Sin embargo, en el caso español se observa que el carácter de las medidas propuestas para la recuperación de las masas de agua subterráneas muy modificadas incluidas en los Planes de Cuenca -en proceso de aprobación-, parecen responder más a la preocupación por la recuperación cuantitativa de estas masas (a través del equilibrio entre extracciones y recargas), que a la recuperación cualitativa de las mismas, provocada en la mayoría de los casos por la contaminación difusa de origen agrario. De este modo, el objetivo establecido por la DMA de reducción progresiva de la contaminación deja de ser prioritario y pasa a ser considerado en la mayoría de los casos, exenciones u objetivos menos rigurosos. Estas decisiones son justificadas por el previsible alto coste que implica la puesta en marcha de las medidas conducentes a alcanzar estos objetivos ambientales y por la dudosa eficacia de las mismas en el medio-largo plazo en acuíferos muy sobreexplotados. No obstante, esta situación no debiera estar justificada en aquellos acuíferos donde el nivel de sobre explotación es moderado o pueda ser subsanado a través de fuentes de suministro alternativas.

Una de las consecuencias de la exención o aplazamiento de los objetivos ambientales es que los costes tanto financieros, como sociales y económicos, ligados a 
la implementación de medidas no llegan a cuantificarse y menos aún, los beneficios ambientales que para la población implica la mejora ambiental de la masa de agua. Ello se justifica en muchos casos, por la falta de consenso en la idoneidad del método a seguir para dicha cuantificación. Sin embargo, desde el ámbito académico se ha avanzado considerablemente en este debate, buscando métodos que permitan la valoración de costes y beneficios ambientales de la forma más rigurosa posible. Así, destacan los basados en la Valoración Contingente (Martín-Ortega, 2009), la Ordenación Contingente (Saz et al., 2009) o el Análisis Multicriterio a través del Proceso Analítico Jerárquico (Mesa et al., 2008). Estas herramientas no sólo permiten estimar los beneficios sociales y ambientales ligados a la puesta en marcha de las medidas, sino que aportan argumentos válidos para justificar su consideración o no en los Planes de la Demarcación afectada.

Sin embargo, los trabajos académicos centrados en valorar explícitamente las medidas conducentes a la mejora cualitativa de las masas de agua son escasos en el caso español. Cabe destacar el trabajo de Martínez y Albiac (2004) en el que se valoran los efectos en la rentabilidad de las explotaciones aragonesas de medidas basadas en la limitación de la dosis de abonado nitrogenado. La mayor parte de los trabajos existentes se focalizan en la gestión del agua del norte y centro de Europa, donde los problemas de contaminación son más acuciantes. Destacan los trabajos de Wustenberghs et al. (2008) y Lacroix et al. (2005) que valoran las medidas destinadas a la reducción de los niveles de contaminación por nitratos en la región de Flandes y en Francia respectivamente. Son varios los trabajos que se centran en analizar la posible incompatibilidad de objetivos ambientales y económicos que supone la recuperación cualitativa de una masa de agua. Para ello Almasri (2007) recurre a modelos multicriterio mientras Fezzi et al. (2010) y Dworak y Pielen (2010) lo hacen bajo un enfoque territorial, minimizando en ambos casos los costes para el sector agrario que implica la mejora global de la calidad del agua. La territorialización de la aplicación de la DMA implicaría la focalización de las políticas a áreas concretas de alto valor ambiental.

Este trabajo trata de llenar el actual vacío existente en lo referente a la valoración de las medidas conducentes a la recuperación cualitativa de una masa de agua desde el enfoque metodológico propuesto por la DMA. A través de una propuesta metodológica aplicada a una masa de agua subterránea muy modificada por alta contaminación por nitratos, se va a tratar de valorar la eficacia de la puesta en marcha de una combinación de medidas que, aplicadas de forma concatenada, conducirían a la recuperación progresiva de la calidad ambiental de sus aguas, una vez que se hayan estabilizado los niveles piezométricos del acuífero en cuestión. En concreto, este trabajo se centra en el acuífero 06.060 que abastece la zona regable del Bajo Almanzora (Almería) y que en la actualidad está sometido a un proceso creciente de sobreexplotación causado por la actividad agraria.

Además, en este ejercicio de simulación se cuantificarán los costes económicos y sociales que implica la puesta en marcha de las medidas y que están relacionados con cambios profundos en la actividad agraria de la zona. En ningún caso este trabajo pretende proponer medidas para su posible incorporación en los planes de cuenca, sino que trata de hacer un ejercicio de prospección sobre la aplicación de los prin- 
cipios de la DMA a una masa concreta, cuantificando posibles efectos en el área de influencia de la zona regable e incluyendo las consideraciones oportunas sobre los efectos ambientales de la continuidad del escenario tendencial.

El artículo se ha organizado del siguiente modo. El epígrafe siguiente se destina a mostrar la metodología propuesta por la DMA basada en al análisis coste-eficacia y que se compone de dos partes bien diferenciadas: la primera destinada a modelizar la presión sobre la masa de los lixiviados procedentes de la fertilización de los cultivos, y la segunda, destinada a explicar la metodología aplicada para cuantificar los costes que implican las diferentes medidas propuestas para la recuperación paulatina de la calidad de las aguas del acuífero. El apartado tercero se destina a mostrar la zona de estudio analizando las fuentes de presión sobre la masa de agua y a presentar las medidas propuestas para su recuperación. El siguiente apartado se destina a mostrar los resultados tras la aplicación de la metodología al caso de estudio. Por último, se presentan algunas conclusiones y reflexiones de interés relativas a la implementación del análisis coste-eficacia.

\section{Metodología}

La metodología seguida para valorar los costes de la recuperación ambiental de la masa de agua objeto de este trabajo será la que propone la DMA. Se trata de establecer en el proceso de planificación un programa de medidas específico para cada objetivo ambiental, de modo que en el proceso de diseño y selección de estas medidas se tengan en cuenta criterios de racionalidad económica (minimización de los costes). Para ello propone el Análisis Coste-Eficacia (ACE en adelante) como método de valoración y selección de las posibles medidas. En el Gráfico 1 aparecen resumidos en forma de esquema los contenidos de la metodología que este trabajo propone.

El ACE supone calcular el índice coste-eficacia, que es el cociente entre el coste anual equivalente y la mejora efectuada con la medida. Para cada aspecto que se pretenda conseguir se seleccionan aquellas medidas con menor índice coste-eficacia que sean suficientes para conseguir el objetivo fijado. La DMA establece que para calcular la eficacia hay que identificar a qué tipo de indicador afectará la medida. En este estudio de caso se recurre a indicadores del estado químico que definen el estado cualitativo de las masas de aguas subterráneas.

Si del análisis de las medidas se evidenciase que los costes necesarios para alcanzar los objetivos ambientales en determinadas masas de agua son superiores a los beneficios obtenidos ("costes desproporcionados" empleando el lenguaje de la DMA), tal circunstancia permitirá justificar una exención en relación al logro del buen estado de la masa (Berbel et al., 2009). Para dichas masas se puede plantear, bien un aplazamiento para el logro del buen estado, o bien la definición de un objetivo menos riguroso. La DMA, a través de la guía Wateco (CIS, 2003)ํㅜㄹ considera tanto las me-

Documento guía para la estrategia común de implementación de la Directiva elaborado por el grupo de trabajo de aspectos económicos (Working Group 2.6 - WATECO). 
didas básicas, cuya inversión ya está recogida en los instrumentos que proporciona la legislación vigente en materia ambiental, como las complementarias, que requieren inversión específica y están presupuestadas en los planes de cuenca y sobre las cuales es preciso realizar el ACE.

\section{GRÁFICO 1}

\section{Esquema de aplicación del análisis Coste-Eficacia}

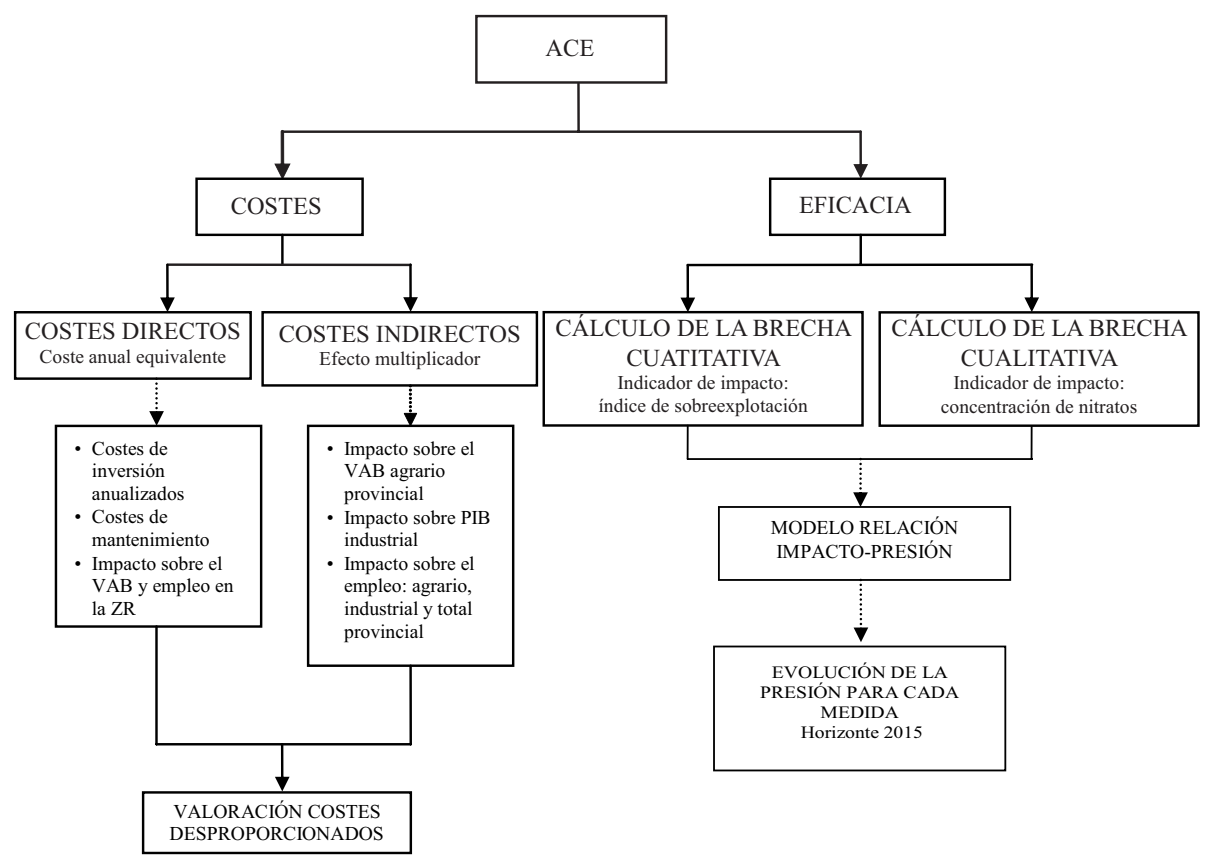

Fuente: Elaboración propia.

\subsection{Cálculo de los costes}

El ACE considera el coste directo de la medida, que incluye los costes de inversión anualizados, el coste de mantenimiento y otros costes económicos que implique la medida. Para el cálculo del Coste Anual Equivalente (CAE), se utiliza la siguiente fórmula:

$$
C A E=I \cdot \alpha n_{i}+C E M+C E S A+C I
$$


Donde $I$ es la inversión requerida, $\alpha n_{i}$, es el factor de amortización anual y CEM es el coste de explotación y mantenimiento. Estos costes se complementan con la introducción de los costes directos de tipo económico, social y ambiental, CESA, y los costes indirectos asociados a la medida, $C I$, que valoran el impacto económico, social y ambiental en una escala espacial superior. En el cálculo de la anualidad deberá tenerse en cuenta, en su caso, la vida útil de todos y cada uno de los elementos necesarios para la ejecución de la medida, el horizonte temporal para el que se realiza el análisis y el plazo de ejecución de la medida hasta su puesta en marcha. Deberá especificarse la tasa de descuento utilizada para el cálculo de la anualidad. El coste de las medidas se valorará a precios constantes indicándose el año de referencia utilizado.

Para la valoración de los costes directos e indirectos de carácter económico y social ligados a la mejora ambiental de una masa de agua, se recurrirá al análisis de los impactos de la medida propuesta en la actividad económica. El análisis llevado a cabo en este trabajo se ha basado en los trabajos previos realizados para la cuenca del Segura por Alcalá y Sánchez (2002), en los que se analizan los efectos que tiene en el sector agrario de la provincia de Murcia la disponibilidad de agua obtenida a través del Trasvase Tajo-Segura. En el caso que aquí se trata, se estimarán los efectos directos e indirectos que tiene la recuperación ambiental de la masa de agua, mediante la valoración de los efectos de la puesta en marcha de las medidas en la actividad agraria y en el empleo agrario de la zona de influencia, que en este caso será tanto la zona regable como la provincia.

Para estudiar el impacto de la actuación es preciso disponer de los valores de algunos parámetros que relacionen las variables que midan los impactos socio-económicos con las variables que cuantifican las presiones sobre los recursos hídricos: efecto en el $\mathrm{VAB}^{2}$ y en el empleo de la zona regable de las variaciones en la gestión de agua (impacto directo), y la repercusión de estos cambios en el total de la actividad agraria, el sector industrial y el conjunto de la economía de la provincia (impactos indirectos). Se trata de medir la variación de la producción agraria en la zona regable y en la provincia de Almería, respecto a la variación de recursos hídricos en la zona regable (Gil et al., 2010). Esta relación se cuantifica a través de la elasticidad del VAB agrario respecto a la disponibilidad hídrica junto con el multiplicador del VAB agrario sobre el VAB provincial, que mide cómo se transmite la variación porcentual de una unidad de VAB en la zona regable y en el VAB de la provincia (ver Cuadro 1).

Un segundo parámetro que se precisa para medir el impacto en términos de empleo es la elasticidad del empleo agrario con respecto al VAB agrario. En este trabajo se ha optado por obtener dicho valor a partir de los datos de la Contabilidad Regional (INE varios años) para el caso de la provincia de Almería, considerando la relación entre la variación porcentual del empleo agrario con la variación porcentual del VAB entre los años 2000 y 2007 (último año para el que se dispone de datos en algunas series). A partir de estos datos, se obtiene la elasticidad del empleo con respecto al VAB provincial. Del mismo modo, se ha obtenido la elasticidad del empleo total

Valor Añadido Bruto: valor de la producción menos el valor de los consumos externos de la explotación. 
provincial respecto al PIB total de la provincia, y la elasticidad del empleo industrial respecto al PIB industrial de la provincia. El valor de dichas elasticidades se muestra en el Cuadro 1.

A la hora de analizar los efectos agregados, esto es, sobre el PIB y el empleo total de la economía de la provincia, se tendrán en cuenta los efectos multiplicadores que ejercen las ramas agrarias sobre el resto de actividades. Éstos se deben a las interrelaciones entre las diferentes actividades como consecuencia de las relaciones de compra y venta de cada uno de los sectores con los restantes. Las tablas InputOutput constituyen un instrumento de partida para la cuantificación de los efectos multiplicadores de las diferentes ramas sobre el conjunto de la economía. La metodología Input-Output permite cuantificar tanto los impactos directos (impacto inicial en el resto de actividades antes de producirse los ajustes en las producciones de los demás sectores) como los indirectos (ajustes en las producciones del resto de ramas de actividad). Lamentablemente, no existe ningún trabajo que aplique esta metodología en la zona de estudio o en la provincia de Almería, por lo que se carece de los valores de los multiplicadores que las diferentes ramas tienen sobre la economía agregada en la zona. Por ello, se ha optado por adoptar los valores de los multiplicadores que algunos trabajos han obtenido para la región andaluza. Para obtener el efecto multiplicador del VAB agrario en el PIB de la provincia se ha recurrido a los trabajos de Rodríguez y Llanes (2004) y Cardenete y Sancho (2003). El multiplicador considerado en este caso es de 1,4. Para ver el efecto multiplicador del VAB agrario en el PIB industrial se ha recurrido al trabajo de Polo et al. (1991). El multiplicador considerado en este caso para la provincia de Almería es de 1 teniendo en cuenta que a nivel nacional el multiplicador obtenido por estos autores es de 1,61.

\section{CUADRO 1}

Parámetros utilizados para el cálculo de los costes

\begin{tabular}{|c|c|c|c|}
\hline & Parámetro & Fuente & Valor \\
\hline \multirow{4}{*}{ Elasticidades } & VAB agrario/disponibilidad hídrica & Gil et al. (2010) & 0,93 \\
\hline & Empleo agrario/VAB Agrario & Contabilidad regional INE (2007) & 0,86 \\
\hline & Empleo total /PIB & Contabilidad regional INE (2007) & 0,98 \\
\hline & Empleo industrial/PIB industrial & Contabilidad regional INE (2007) & 0,60 \\
\hline \multirow{2}{*}{ Multiplicadores } & VAB agrario/VAB & $\begin{array}{l}\text { Rodríguez y Llanes (2004), Cardenete } \\
\text { y Sancho (2003) }\end{array}$ & 1,40 \\
\hline & VAB agrario/VAB industrial & Polo et al. (1991) & 1,00 \\
\hline
\end{tabular}

Fuente: Elaboración propia a partir de las referencias citadas. 


\subsection{Análisis de la eficacia}

Siguiendo la guía Wateco (CIS, 2003), para evaluar la eficacia de las medidas se parte de la evaluación del estado de las masas de agua correspondiente al escenario tendencial, y se compara con su "situación con medida". La diferencia que exista entre ambos estados es el grado de eficacia de la medida.

El indicador del estado de la masa puede obtenerse de dos formas: mediante la reducción de las presiones significativas que sufren, es decir, reducción de la magnitud de las presiones reflejadas en el inventario de presiones ${ }^{3} ; \mathrm{o}$, mediante la reducción de los impactos medidos en las masas de agua, es decir, la mejora en los indicadores de estado de las masas de agua que propicia la medida. Este método de evaluación requiere conocer previamente los efectos de la medida en la reducción de presiones y la susceptibilidad de las masas de agua ante estas presiones. Es en este último enfoque en el que se va a centrar el análisis de la eficacia de las medidas propuestas en este trabajo. En este caso, se parte de una conjunción de medidas destinadas a la recuperación del acuífero mediante la puesta en marcha de infraestructuras y nuevos modos de gestión del agua en la sub-cuenca, aspectos que serán detallados en la descripción de las medidas del apartado siguiente. Se trata de valorar la eficacia de las medidas conducentes a la recuperación del estado cualitativo de las aguas, una que vez que se ha alcanzado el equilibrio en los niveles piezométricos mediante la paralización de las extracciones del acuífero.

El objetivo ambiental que se persigue es alcanzar el nivel mínimo de contaminación de nitratos que establece la Directiva de Nitratos (Directiva 91/676/CEE) relativa a la protección de las aguas contra la contaminación producida por los nitratos

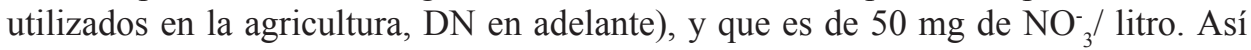
se calcula la brecha cualitativa como la diferencia entre el estado alcanzado con la medida analizada y el objetivo previsto que establece la DN. Para valorar la brecha es preciso conocer la relación que existe entre las distintas medidas y el indicador que mide el estado de la masa. En este trabajo se propone el desarrollo de un modelo que vincule la evolución de la concentración de nitratos en el acuífero con el factor que explica la presión sobre la masa, que en este caso serían las cantidades de nitrógeno lixiviado. Este modelo permite simular su evolución tras la puesta en marcha de la medida.

En primer lugar es necesario conocer cómo es la dinámica del factor de contaminación del acuífero: la contaminación difusa procedente de la actividad agraria en el área de influencia del acuífero. Para ello se ha recurrido a modelos que simulan el balance de nitrógeno en el cultivo siguiendo los criterios y datos del extinto Ministerio de Medio Ambiente y Medio Rural y Marino (MARM, 2008) en su informe sobre el balance de nitrógeno de la agricultura española. Este balance se basa en el cálculo de extracciones de nitrógeno proveniente de la fertilización mineral de todos los cultivos con presencia en la agricultura española, de la fertilización orgánica y de la actividad

3 El inventario de presiones se refiere al conjunto de acciones antropogénicas que afectan al estado ambiental de la masa de agua. Las correspondientes a la masa de agua analizada se encuentran en el Anejo VII del Borrador del Proyecto del Plan Hidrológico de la Demarcación Hidrográfica de las Cuencas Mediterráneas Andaluzas. 
ganadera. Se trata por tanto de obtener las cantidades totales de nitrógeno lixiviadas al acuífero $\left(L_{p}\right)$ procedentes de la zona regable, en $\mathrm{kg}$ para cada año $t$, para cada uno de los cultivos presentes en la zona regable $i$. Este balance se puede expresar como:

$$
L_{t}=\Sigma E_{i t} X_{i t}-\Sigma E x_{i t} X_{i t}
$$

Siendo $E_{i t}$ las entradas de nitrógeno del cultivo $i$ en el período $t$. $E x_{i t}$ son las extracciones de nitrógeno para el mismo cultivo y período. $X_{i t}$ es la superficie dedicada a cada cultivo en cada período. Las entradas de nitrógeno por cultivos y unidad de superficie quedan reflejadas en la ecuación [3].

$$
E_{i t}=D n_{i}+N A_{t-1} \alpha_{i} D_{i}+E_{i t-1} \beta_{i}
$$

$D n_{i}$ son las aportaciones de nitrógeno al cultivo a través de la fertilización mineral. Este dato se ha obtenido para cada cultivo en base al informe realizado por el MARM, en el que se han estimado las dosis que se están aplicando en cada zona de España basadas en encuestas de campo. $N A_{t-1}$ es la concentración de nitratos que existe en la masa de agua, medida en $\mathrm{mg}$ de $\mathrm{NO}_{3}^{-} / 1$, correspondiente al período anterior. Esta concentración se corrige con un factor que depende del cultivo $\left(\alpha_{i}\right)$ y se multiplica por la dosis de riego para cada uno de ellos $\left(D_{i}\right)$. Para el cálculo de las entradas de nitrógeno habrá que tener en cuenta también el nitrógeno residual en el suelo, que equivaldrá a las entradas de nitrógeno en el cultivo en el período anterior $\left(E_{i t-1}\right)$, multiplicado por un factor que representa el porcentaje de cultivo que se transforma en residuo y que queda en el suelo, y que será diferente para cada cultivo $\left(\beta_{i}\right)$. Las extracciones de nitrógeno por cultivo y superficie se establecen según la ecuación [4].

$$
E x_{i t}=Y_{i t} X_{i t} c e_{i}
$$

Las extracciones dependerán de los rendimientos del cultivo en cada período $\left(Y_{i t}\right)$, de la superficie dedicada al mismo $\left(X_{i t}\right)$ y de un coeficiente de extracción de nitrógeno $\left(c e_{i}\right)$ que se establece para cada cultivo y que depende de los rendimientos máximos y del porcentaje de cultivo que se cosecha (Urbano, 1995).

La siguiente fase para estimar la eficacia de cada medida a valorar es conocer la relación que existe entre la evolución del indicador de impacto en el acuífero (mg $\mathrm{NO}_{3}^{-} /$litro) y la evolución de la fuente de presión sobre la masa, que en este caso es el volumen de nitrógeno que se lixivia y que acaba en el acuífero. Como se ha visto en las ecuaciones presentadas anteriormente, la cantidad lixiviada dependerá indirectamente de una serie de variables que se verán modificadas tras la aplicación de cada medida, como pueda ser una variación de la dosis de abonado, de la superficie regada o de la cantidad de nitratos aportada en el agua de riego. Se trata en definitiva, de estimar la contaminación de nitratos en el acuífero partir de la función que expresa la ecuación [5], donde $f\left(L_{t}\right)$ es una función que depende de los cantidades lixiviada y $\varepsilon_{t}$ es el error cuadrático que sigue una distribución normal. 


$$
N A_{t}=f\left(L_{t}\right)+\varepsilon_{t}
$$

Se ha construido un modelo de estimación lineal a partir del ajuste de una serie histórica de datos de concentración de nitratos en la masa analizada (años 19952008) que permite estimar las cantidades lixiviadas. De este modo se dispone de un instrumento que permite evaluar cuál es la eficacia de cada medida. Proyectando las estimaciones del modelo es posible conocer la reducción de la brecha cualitativa en el horizonte 2015 que implica cada medida. Para ello se proyecta en el tiempo la tendencia de los valores de contaminación estimados a partir del modelo, y se compara con el objetivo ambiental perseguido, que sería alcanzar una concentración límite de $50 \mathrm{mg} \mathrm{NO}_{3}^{-} / 1$.

\section{El caso de estudio}

La situación planteada en la introducción para el regadío intensivo del levante español se puede proyectar al caso de estudio de esta investigación. La zona regable que se analiza en este trabajo es Cuevas de Almanzora localizada en el levante almeriense. Tiene una superficie regable de 3.664 ha de las que sólo el $58 \%$ se riegan de forma efectiva, destinándose esta superficie al cultivo de hortícolas al aire libre y cítricos, regados mayoritariamente con riego por goteo. La demanda anual de la Zona Regable según los datos aportados por el Borrador del Plan Hidrológico de las Cuencas Mediterráneas Andaluzas (BPHCMA en adelante), es de 16,22 $\mathrm{hm}^{3}$ al año, siendo el consumo bruto anual de $14,70 \mathrm{hm}^{3}$. Se trata por tanto de un área infradotada con un déficit anual de $1,52 \mathrm{hm}^{3}$.

Este déficit estructural de recursos en la zona se explica por la evolución de los cultivos en los últimos 12 años. Los cultivos hortícolas cultivados de forma intensiva a cielo abierto representan el $42 \%$, frente a la presencia de los cítricos que es del $36 \%$. Éstos han experimentado en los últimos años un incremento notable especialmente debido al incremento del cultivo del mandarino. En el Gráfico 2 se presenta la evolución de los principales cultivos en estos años.

La orientación hacía cultivos más demandantes de nitrógeno como los cítricos (Urbano, 1995), ha provocado un incremento de la contaminación difusa proveniente de la fertilización, lo que tiene una traducción directa en un aumento progresivo de la contaminación por nitratos en el acuífero. La masa afectada en este caso es el acuífero 06.060 denominado "Bajo Almanzora", perteneciente al subsistema V-II de la cuenca del rio Almanzora que a su vez pertenece a la demarcación Cuencas Mediterráneas Andaluzas. 


\section{GRÁFICO 2}

\section{Evolución de la superficie de los principales cultivos en la ZR Cuevas de Almanzora (en hectáreas)}

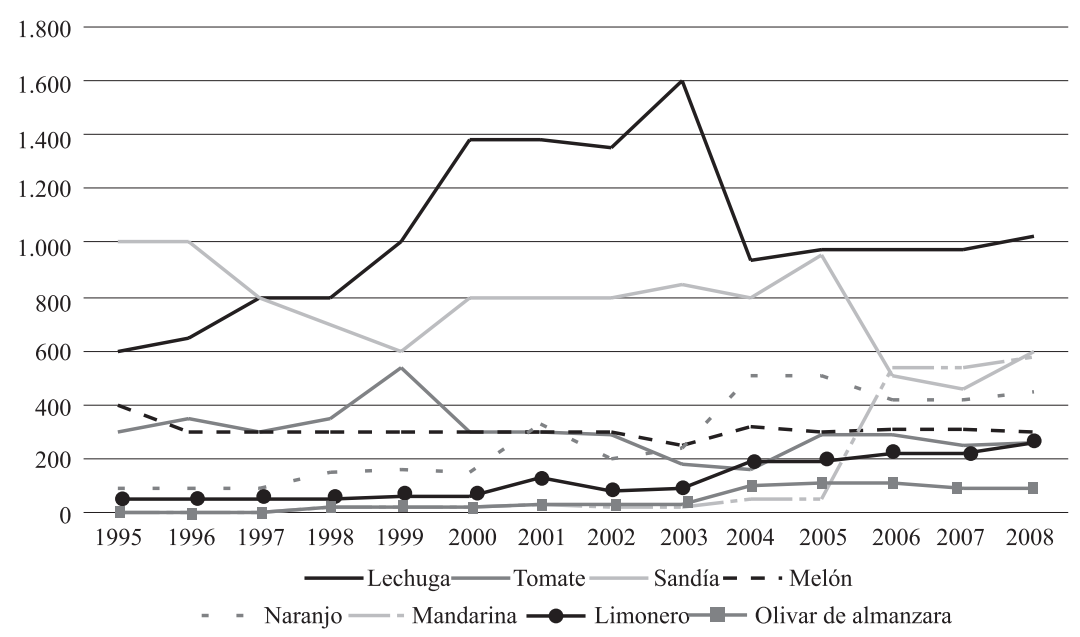

Fuente: MARM - Informes 1T a nivel municipal, varios años.

Esta masa soporta la dinámica del regadío intensivo antes descrita. Así, los datos del BPHCMA muestran que ésta se encuentra sobreexplotada en una cantidad de $0,28 \mathrm{hm}^{3} /$ año. La tendencia proyectada para el año 2015 , tal y como establece el BPHCMA, es que, de no aplicarse medidas, se agravará la situación actual debido al aumento de las extracciones hasta $\operatorname{los} 2,50 \mathrm{hm}^{3}$, siendo el índice de sobreexplotación de $1,14 \mathrm{hm}^{3} /$ año. Esta situación implica una fuerte presión sobre la calidad del agua de la masa. Así en el año 2009 y según la misma fuente, la concentración de nitratos fue de $205 \mathrm{mg} \mathrm{NO}_{3}^{-} / 1$ superando con creces el límite máximo de $50 \mathrm{mg} \mathrm{NO}_{3}^{-/ 1}$ establecido por la DN. Además se están superando los valores umbrales de otros contaminantes como amonio, cloruros o sulfatos. Con estos datos, y teniendo en cuenta la evolución de las presiones sobre la masa, el BPHCMA estima que el estado tanto cualitativo como cuantitativo de la masa para el año 2015 se podría calificar como "muy malo". El Gráfico 3 muestra la evolución de la concentración de nitratos en el acuífero 06.060. Considerando una prolongación de la tendencia actual al año 2015, se proyectan unos niveles de contaminación de $280 \mathrm{mg}$ de $\mathrm{NO}_{3}^{-} / 1$.

Como se puede ver la solución de los problemas de contaminación del acuífero pasa por resolver los problemas ligados a la sobreexplotación del mismo. Éstos tienen su origen tanto en el uso intensivo de los recursos como en un déficit estructural de la cuenca del río Almanzora, donde se ubica la zona regable y la masa de agua analizada. Así, las aportaciones de las masas subterráneas, que son $15 \mathrm{hm}^{3}$ (de los que $8 \mathrm{hm}^{3}$ proceden de la sobreexplotación del acuífero), junto con el agua procedente del 
trasvase Negratín-Almanzora, actualmente en explotación $\left(27,62 \mathrm{hm}^{3}\right)$, no son capaces de satisfacer la demanda de la cuenca, estimada en $63 \mathrm{hm}^{3}$. Este balance mejorará tras la puesta en marcha de las medidas que considera el BPHCMA. Por un lado se cuenta con los aportes de la desaladora del "Bajo Almanzora", proyectada en el marco del programa A.G.U.A del MARM para sustituir las extraciones de las masas subterráneas sobre explotadas. Su puesta en marcha supondrá unos recursos adicionales de $15 \mathrm{hm}^{3}$. Por otra, los recursos provenientes del trasvase del Negratín se completan con la compra voluntaria de derechos de uso de aguas por la empresa Aguas de Almanzora S.A encargada del suministro de la cuenca (tal y como se hizo durante los años 2005-2008 declarados como secos por el MARM). Estas compras suponen unos aportes adicionales de 11,8 hm³ (Calatrava y Gómez-Ramos, 2009; Sánchez-Picón, 2011). Suponiendo un escenario de flexibilización de las cesiones no restringidas únicamente a períodos de sequía declarada -teniendo en cuenta las condiciones que establece la norma que regula el trasvase Negratín-Almanzora, y contando con los recursos aportados que contempla el programa de medidas del BPHCMA- antes mencionado, se podría satisfacer las demandas de la Cuenca, pues se alcanzarían unos recursos disponibles totales de 69,4 hm³ (Olmedilla y Gómez-Ramos, 2011).

Esta nueva situación propiciaría la recuperación de los niveles de los acuíferos de la cuenca y, posteriormente, tal y como establece la DMA, sería posible la recuperación de la calidad de sus aguas. Este plausible escenario plantea la necesidad que este trabajo aborda, y que no es otra que profundizar en las medidas conducentes a la recuperación cualitativa de las masas de agua afectadas de este subsistema, una vez solucionados los problemas de déficit.

\section{GRÁFICO 3}

\section{Evolución de la contaminación por nitratos en el acuífero Bajo Almanzora y proyección al año 2015 (en mg $\mathrm{NO}_{3}^{-} / \mathrm{l}$ )}

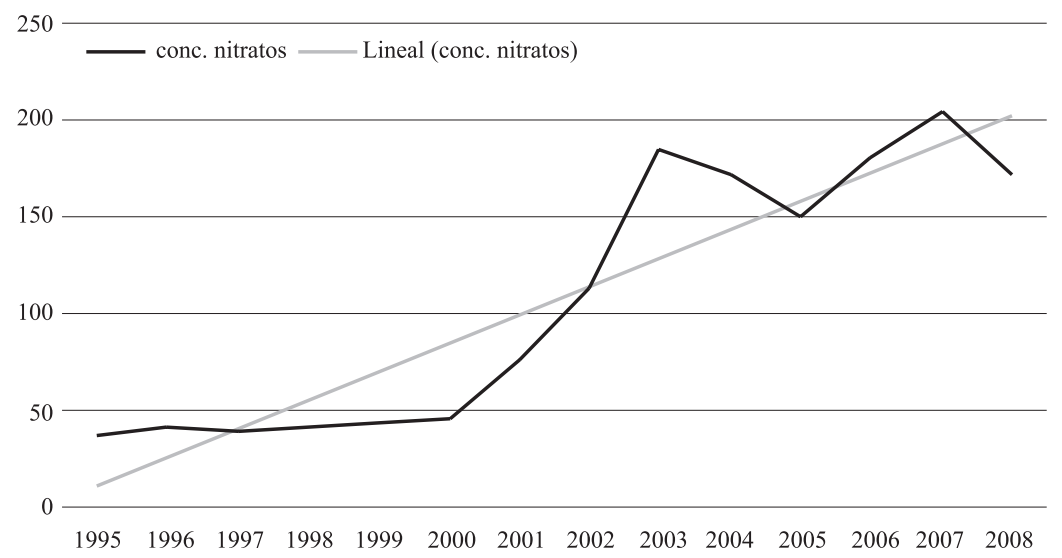

Fuente: Elaboración propia con datos del BPHCMA. 
Las medidas que se proponen para la recuperación cualitativa del acuífero parten del supuesto de que en la cuenca se satisfacen las demandas y que los acuíferos de la cuenca no se explotarán más a raíz de la puesta en marcha de la desaladora del Bajo Almanzora, el trasvase y la liberalización de las cesiones. Todo ello en un contexto de gobernanza en la gestión de los recursos que impida las extracciones.

\section{Medida Básica: aplicación DN}

Como medida básica se plantea aquella que es consecuencia de la aplicación de la normativa vigente, en este caso, la DN, que obliga a realizar un plan de actuación en aquellas zonas declaradas como vulnerables por el Estado Miembro. La zona correspondiente al caso de estudio fue declarada como sensible en 2005 por la Junta de Andalucía (Valle del Almanzora- Decreto 204/2005, de 27 de septiembre). El Plan de Actuación declarado para la zona sensible implica la aplicación de un código de buenas prácticas basadas en el establecimiento de dosis máximas de abonado nitrogenado. El plan de actuación para esta zona vulnerable establece una dosis máxima recomendada de $210 \mathrm{~kg}$ de $\mathrm{N} / \mathrm{ha}$.

\section{Medida 1: Riego con agua desalada y trasvasada}

Tras la puesta en marcha de la desaladora y la incorporación de los recursos procedentes del trasvase y compra de derechos, se supone que los lixiviados de nitratos procedentes del agua de riego disminuirán progresivamente al regar con agua libre de sustancias contaminantes. Anteriormente se producía un efecto de retroalimentación al regar con agua procedente del acuífero con una alta concentración de nitratos. A través de esta medida se tratará de ver cómo es la mejora de los niveles de concentración de nitratos si la zona regable se riega con agua libre de nitratos procedente de fuentes de suministro superficiales, que será agua desalada y de la cuenca alta del Guadalquivir.

\section{Medida 2: Aplicación de un programa de fertirrigación}

El Gráfico 4 muestra cómo ha sido la evolución de la contaminación por nitratos en el acuífero y la evolución de la superficie destinada a los cítricos en la ZR de Cuevas de Almanzora según los datos del MARM a nivel municipal (hojas 1T varios años). Como se puede apreciar estas variables han seguido una evolución similar (coeficiente de correlación de Pearson 0,89), por lo que se puede deducir que estos cultivos han influido en el progresivo aumento de la contaminación en la zona ya que la superficie destinada al resto de cultivos se ha mantenido estable en estos años. Para actuar sobre el foco del problema se propone como medida un programa lanzado desde las administraciones que incentive la introducción de sistemas de fertirrigación para cítricos, de modo que se aplique de forma puntual la dosis de abonado adaptada a las necesidades del cultivo en cada momento. A través de este programa se estima que se podría disminuir la dosis de nitrógeno aplicada al cultivo en un 25\% (Cadahía, 2005). 
Este tipo de iniciativas podrían enmarcarse en el actual Programa de Desarrollo Rural (PDR) de la Comunidad Autónoma Andaluza (2007-2013), dentro de las medidas enmarcadas en el eje 1 ligadas a la modernización de explotaciones, entre cuyos objetivos se encuentran potenciar un uso y gestión sostenible del agua de riego, así como del resto de medios de producción y la lucha contra la contaminación difusa de la agricultura (Junta de Andalucía, 2006). Los Programas Operativos llevados a cabo por las Organizaciones de Productores en el marco de la Organización Común de Mercados de Frutas y Hortalizas también contemplan la subvención de este tipo de programas. En cualquier caso la subvención sería igualmente del 50\% de la inversión con fondos bien del FEAGA o bien del FEADER.

\section{GRÁFICO 4}

\section{Evolución de la contaminación del acuífero y de la superficie destinada a cítricos}

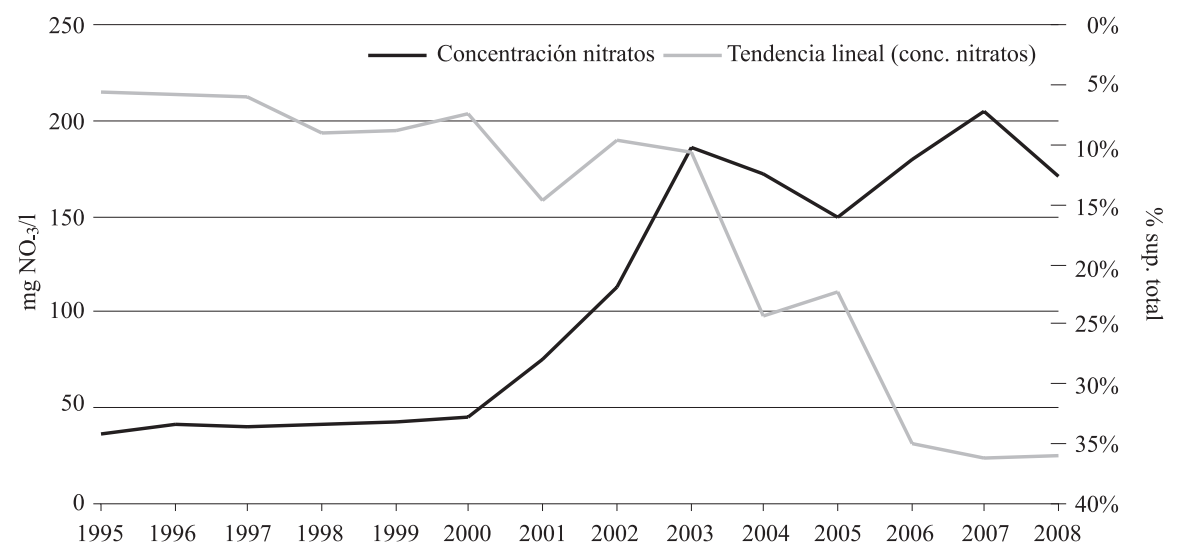

Fuente: Elaboración propia con datos del MARM (hojas 1T varios años).

\section{Medida 3: Eliminación de cultivos}

Esta medida se plantea como última opción para alcanzar la total recuperación de la masa de agua. Se trata de captar el efecto individualizado en la contaminación de los principales grupos de cultivos: hortícolas y cítricos. Los costes que implicaría la medida están relacionados con el efecto multiplicador provocado en la economía de la provincia por un cambio en el modelo productivo del área como consecuencia del abandono parcial de la agricultura intensiva de regadío. La magnitud final de esta cifra en relación con los beneficios generados podría suponer un coste desproporcionado, que en su caso podría conducir a la exención total o parcial en la consecución de los objetivos. 


\section{Análisis de resultados}

Los modelos reflejados en el Cuadro 2 que ajustan la ecuación [5] para cada una de las medidas, se han construido a partir de series de concentraciones anuales de nitratos en el acuífero 06.060 Bajo Almanzora, entre los años 1995-2008 suministrados por la Agencia Andaluza del Agua y recogidos en el BPHCMA. El cálculo de las cantidades lixiviadas se ha realizado con los datos de evolución de las superficies de cultivos en los años de la serie, datos procedentes del MARM en sus informes 1T a nivel municipal. Los datos de rendimientos anuales de cultivos proceden de los Anuarios de Estadística Agraria del Ministerio de Medio Ambiente Medio Rural y Medio Marino (MARM, varios años) para los años de la serie. La variable dummy representa el cambio de tendencia acaecido en el acuífero en la evolución de la variable explicativa y que viene determinada por un aumento importante de la superficie destinada a cítricos en el año 2002 (ver Gráfico 3).

Los resultados obtenidos tras la simulación de las medidas planteadas se recogen en el Cuadro 3 y en el Gráfico 5. En el Cuadro 3 se ha presentado la reducción de las cantidades de nitrógeno lixiviadas al suelo que implica la aplicación concatenada de medidas, de forma que los logros conseguidos por una se suman a la siguiente. También se muestran, mediante la proyección de la tendencia a través de un ajuste econométrico, los niveles de concentración de nitratos alcanzados en la masa de agua en el horizonte 2015 para cada medida (ver Gráfico 6). La última columna del Cuadro 3 muestra la reducción de la brecha, considerando ésta como la diferencia entre los niveles de contaminación alcanzados con la medida en el horizonte 2015 y los niveles objetivos, que como se ha dicho se sitúan en $50 \mathrm{mg} \mathrm{NO}_{3}^{-} / 1$.

\section{CUADRO 2}

\section{Especificación de los modelos presión-impacto para cada medida}

\begin{tabular}{lccccc}
\hline \multirow{2}{*}{ MEDIDA } & \multicolumn{2}{c}{ Especificación modelo } & \multicolumn{2}{c}{ Estadísticos } \\
\cline { 2 - 6 } & cte & $\operatorname{coef}(L)$ & dummy & rho (corr) & $F$ \\
\hline Medida básica cumplimiento Directiva Nitratos & $-7,13(0,78)$ & $8,43(0,04)$ & $75,71(0,007)$ & 0,90 & 51,55 \\
\hline Desaladora + trasvase & $-47,60(0,38)$ & $0,001(0,09)$ & $91,81(0,001)$ & 0,88 & 66,95 \\
\hline Programa fertirrigación & $-14,41(0,85)$ & $0,001(0,44)$ & $108,51(0,000)$ & 0,85 & 33,75 \\
\hline Eliminación cítricos & $-11,67(0,33)$ & $0,001(0,82)$ & $119,55(0,000)$ & 0,84 & 30,84 \\
\hline Eliminación hortícolas & $-33,23(0,001)$ & $0,001(0,05)$ & $92,27(0,000)$ & 0,89 & 49,41 \\
\hline Eliminación cítricos + hortícolas & $-44,06(0,001)$ & $0,002(0,225)$ & $97,97(0,001)$ & 0,86 & 36,88 \\
\hline
\end{tabular}

Entre paréntesis $t$ de Student.

Fuente: Elaboración propia. 


\section{CUADRO 3}

\section{Eficacia de las medidas}

\begin{tabular}{|c|c|c|c|c|}
\hline \multicolumn{2}{|c|}{ Escenario } & $\begin{array}{c}\text { Reducción lixiviados } \\
\text { respecto esc. tendencial } \\
(\%)\end{array}$ & $\begin{array}{c}\text { Concentración nitratos } \\
\left(\left(\mathrm{mg} \mathrm{NO}_{3}^{-} / \mathrm{l}\right)\right.\end{array}$ & $\begin{array}{c}\text { Reducción Brecha } \\
(\%)\end{array}$ \\
\hline \multicolumn{2}{|l|}{ Tendencial } & - & 295 & - \\
\hline \multicolumn{2}{|c|}{ Aplicación Directiva Nitratos } & 4 & 213 & 13 \\
\hline \multicolumn{2}{|c|}{ Desaladora + trasvase } & 47 & 163 & 53 \\
\hline \multicolumn{2}{|c|}{ Programa fertirrigación } & 58 & 137 & 64 \\
\hline \multirow{3}{*}{$\begin{array}{l}\text { Eliminación } \\
\text { superficie regada }\end{array}$} & Cítricos & 69 & 100 & 79 \\
\hline & Hortícolas & 89 & 90 & 83 \\
\hline & Cítricos+hortícolas & 99 & 10 & 116 \\
\hline
\end{tabular}

Fuente: Elaboración propia.

\section{GRÁFICO 5}

\section{Evolución de la contaminación en el horizonte 2015 para el escenario tendencial y cada una de las medidas (en $\mathrm{mg} \mathrm{NO}_{3}^{-} / \mathrm{l}$ )}

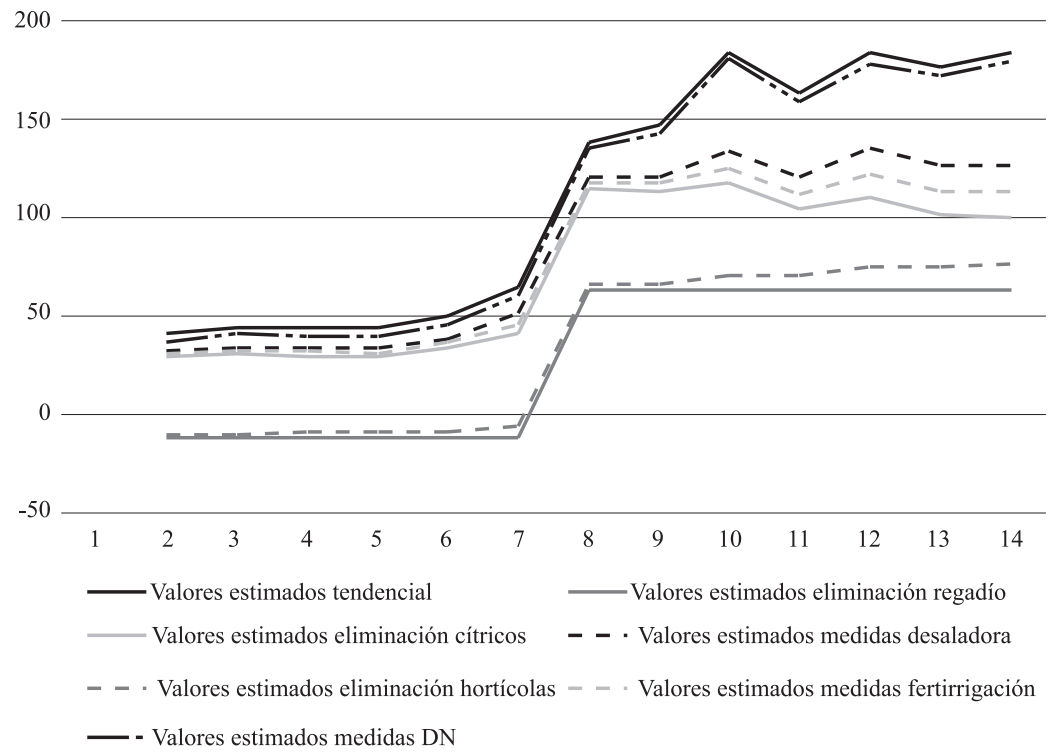

Fuente: Elaboración propia. 


\section{Medida básica: aplicación de la DN}

Para esta medida se ha considerado una reducción de la dosis de abonado hasta el nivel máximo establecido para este área, que es de $210 \mathrm{~kg}$ de N/ha. Siguiendo la metodología de cálculo del balance de nitrógeno (ecuación [1]) se alcanza una reducción estimada del 4\% de las cantidades lixiviadas respecto al escenario tendencial. La simulación de estos datos a través de la ecuación [5] refleja una reducción estimada de la contaminación final en el acuífero del 2\% respecto al escenario tendencial. La proyección para el año 2015 permite estimar una concentración de $263 \mathrm{mg} \mathrm{NO}_{3}^{-} / 1$, lo que supone una reducción de la brecha en este escenario del 13\% (ver Cuadro 3). Esta medida, al tratarse de una medida básica, se realiza sin ningún tipo de inversión por lo que su coste es cero. Se ha considerado que una disminución en la dosis no implica una reducción de los rendimientos.

\section{Medida 1: Riego con agua procedente de la desaladora y del trasvase}

La puesta en marcha de la desaladora y la utilización del agua del trasvase (a través de la concesión y de la compra de derechos), supone que la entrada de nitrógeno en el cultivo a través del agua de riego disminuya, de forma que los lixiviados se reducen en un 5\%. Suponiendo que los regantes continúan cumpliendo con las limitaciones establecidas en la $\mathrm{DN}$, los resultados alcanzados con la aplicación de esta medida se sumarían a los obtenidos con la medida básica. La reducción acumulada de la brecha se estima en un 53\%, siendo la concentración de nitratos en 2015 de 163 $\mathrm{mg} \mathrm{NO}_{3}^{-/ 1}$ (ver Cuadro 3). De estos resultados se deduce que una gestión del acuífero basada en la gobernanza implicaría una reducción sustancial de los niveles de contaminación en el acuífero.

El coste directo de esta medida dependerá de los costes de inversión y mantenimiento de la desaladora y de las obras del trasvase, así como del coste anual que implica el sobrecoste que deberán pagar por el agua los regantes para adquirir el agua procedente del trasvase, de la compra de derechos y de la desaladora. En el Cuadro 6 aparece el coste directo de las inversiones que conlleva la adquisición de nuevos aportes procedentes de estas fuentes de suministro. Olmedilla y Gómez-Ramos (2011) estimaron un CAE total para esta medida de 31 millones de euros al año, teniendo en cuenta el precio que los regantes deberán pagar por el agua suministrada, que será de $0,50 € / \mathrm{m}^{3}$ para el agua trasvasada, $0,43 € / \mathrm{m}^{3}$ por el agua desalada y una media de $0,68 € / \mathrm{m}^{3}$ por el agua comprada. El coste indirecto de esta medida se centra fundamentalmente en los efectos del trasvase y las cesiones de aguas en la cuenca cedente, y se cuantificaría conociendo los impactos económicos, sociales y ambientales que supone la renuncia a parte de su dotación. La estimación de estos efectos es de suma complejidad y no es objeto de este estudio.

\section{Medida 2: Programa de fertirrigación para citricos}

Unida a los efectos de las medidas anteriores, esta medida supone una reducción de las cantidades lixiviadas respecto al escenario tendencial del $58 \%$, lo que implica una reducción de la brecha del 64\% alcanzando una contaminación en el acuífero de 
$137 \mathrm{mg} \mathrm{NO}_{3}^{-/ 1}$ (ver Cuadro 3). Para valorar el coste de la medida se considera que el $100 \%$ de estos árboles se riegan con sistemas localizados (Junta de Andalucía, 2008). A partir de la información obtenida de los datos recabados a través de la consulta de presupuestos de proyectos similares, se ha estimado que el coste de la inversión es de $2.000 € /$ ha. Se considera que la infraestructura en tuberías y cabezal de riego ya existe, teniendo que asumir únicamente la inversión correspondiente a los sistemas de depósitos, programación y bombeo. Se asume que la iniciativa es acogida de forma mayoritaria ya que los programas operativos y/o el PDR podrían financiar el 50\% de la inversión. El coste estimado de la medida será de 4,3 millones de euros para las 1.280 has de cítricos afectadas (Junta de Andalucía, 2008). Este importe incluye tanto la inversión pública como privada. Para completar la valoración de esta medida habría que tener en cuenta la disminución de los costes anuales de producción por hectárea debido a la diminución del $20 \%$ de la dosis de abono nitrogenado. Se han considerado los estudios de costes realizados por el MARM en las explotaciones de cítricos de Andalucía para el año 2010 (MARM, 2010). Teniendo en cuenta la distribución de cultivos en la ZR, se ha estimado que esta reducción de costes implica una reducción global de los costes en la zona del 17\%. Ello se traduce en un aumento del margen neto para toda la ZR, que implica un aumento del VAB de la misma valorado en 0,62 millones de euros. Para el cálculo del CAE de esta medida se ha estimado una vida útil de la inversión de 10 años (ver Cuadro 5).

\section{Medida 3: eliminación de cultivos}

El último bloque de medidas se plantea ante el hecho de que con la aplicación conjunta de las medidas anteriores no se alcanza el objetivo de reducción de contaminación en el acuífero, es decir, obtener niveles inferiores a $50 \mathrm{mg} \mathrm{NO}_{3}^{-} / 1$ (ver Cuadro 3). Esta medida es más radical ya que supone la reducción de una parte de la superficie de riego. La medida se plantea a partir de la consideración del efecto de la eliminación de los cultivos hortícolas frente a los cítricos, dada su diferente rentabilidad y capacidad contaminante. En el Gráfico 6 se muestra la simulación de las cantidades de nitrógeno lixiviadas por cada grupo de cultivos presentes en la ZR. Como se observa, son los cítricos los que en mayor medida han contribuido a incrementar en los últimos años las cantidades lixiviadas en la zona regable, aunque en términos absolutos los hortícolas aportan más lixiviados por representar más del $60 \%$ de la superficie regada. 


\section{GRÁFICO 6}

\section{Evolución estimada de las cantidades lixiviadas de los grupos de cultivos}

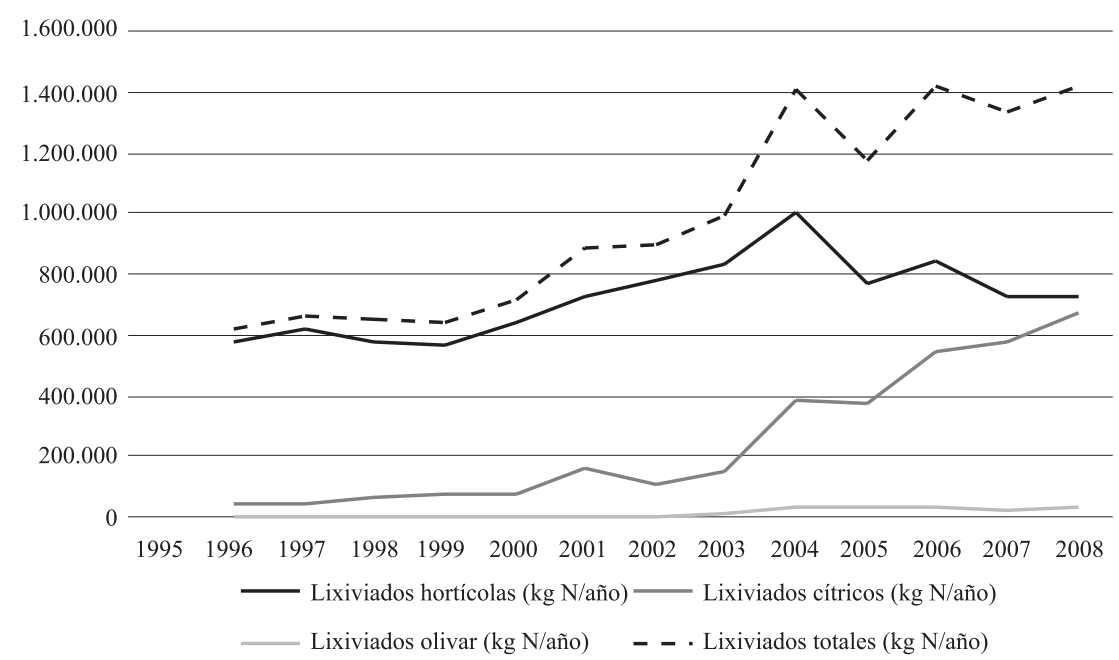

Fuente: Elaboración propia.

Como se muestra en el Cuadro 3, la eliminación de los hortícolas supone una reducción de las cantidades lixiviadas del 89\%, mientras que la eliminación de los cítricos supone un $69 \%$, si bien estos últimos representan un 36\% de la superficie de la ZR. Los niveles de contaminación que se alcanzarían serían de 90 y de 100 $\mathrm{mg}$ de $\mathrm{NO}_{3} / 1$ respectivamente, siendo la reducción de la brecha en el horizonte 2015 de alrededor del $80 \%$ en ambos casos. Dado que con ninguna de las dos medidas se alcanza el objetivo ambiental perseguido, se ha simulado una situación en los que se eliminarían ambos grupos de cultivos. En este caso, la reducción de lixiviados alcanzaría el 99\% y los niveles de contaminación alcanzados en el horizonte 2015 serían de $10 \mathrm{mg}$ de $\mathrm{NO}_{3}^{-} / 1$.

Los costes de aplicación de la medida equivalen, por un lado a los costes directos sobre el VAB y el empleo de la ZR y por otro, a los costes indirectos sobre el PIB y el empleo total e industrial de la provincia que supone la eliminación de estos cultivos. Para el primer caso, y en base a los datos de superficie y margen bruto por hectárea que aportan la Junta de Andalucía (2008) y el MARM (2010), se estima que la perdida de VAB por la eliminación de 1.280 has de cítricos en la ZR sería de 2,89 millones de euros, mientras que la desaparición de 2.175 has de hortícolas supondría una pérdida en el VAB de la ZR de 15,84 millones de euros (considerando los precios de 2009). La pérdida de jornales es mayor con la eliminación de hortícolas, siendo del 77\% respecto a la situación de 2009 (ver Cuadro 4). 


\section{CUADRO 4}

\section{Costes directos e indirectos de la eliminación de cultivos hortícolas y cítricos}

\begin{tabular}{|c|c|c|c|c|c|}
\hline Área de influencia & Indicador & $\begin{array}{c}\text { Situación } \\
\text { actual }\end{array}$ & $\begin{array}{c}\text { Eliminación } \\
\text { hortícolas }\end{array}$ & $\begin{array}{l}\text { Eliminación } \\
\text { cítricos }\end{array}$ & $\begin{array}{c}\text { Eliminación } \\
\text { ambas }\end{array}$ \\
\hline \multirow{2}{*}{ Z.R. Cuevas Almanzora } & VAB (mill. €) & 18,36 & $2,89(84 \%)$ & $15,54(15 \%)$ & $0,150(99 \%)$ \\
\hline & Empleo (mil. pers) & 0,32 & $0,038(77 \%)$ & $0,132(22 \%)$ & $0,001(99 \%)$ \\
\hline \multirow{2}{*}{ Sector agrario Almería } & VAB agr. (mill. $€$ ) & $1.210,00$ & $1.195(1,2 \%)$ & $1.207(0,2 \%)$ & $1.192(1,5 \%)$ \\
\hline & Empl.agr.(mil. pers.) & 46,60 & $46(2,8 \%)$ & $46,5(0,08 \%)$ & $45,99(3,6 \%)$ \\
\hline \multirow{2}{*}{ Sector industrial Almería } & PIB Ind. (mill. €) & 54,70 & $54,0(1,2 \%)$ & $54,6(0,2 \%)$ & $53,9(1,5 \%)$ \\
\hline & Empl. ind. (mil. pers) & 18,00 & $17,84(0,8 \%)$ & $17,97(0,1 \%)$ & $17,81(1,0 \%)$ \\
\hline \multirow{2}{*}{ Economía Almería } & PIB tot (mill. €) & $13.871,00$ & $13.623(1,7 \%)$ & $13.826(0,3 \%)$ & $13.579(2,1 \%)$ \\
\hline & Empl. Tot (mil. pers) & 312,00 & $306(1,7 \%)$ & $311(0,3 \%)$ & $305(2,0 \%)$ \\
\hline
\end{tabular}

Fuente: Elaboración propia.

Los efectos de la medida en el VAB y empleo agrario de la provincia se difuminan mucho, ya que la ZR sólo representa el 5\% de la superficie regable de la provincia. Como se observa en el Cuadro 4, los efectos, aunque pequeños, son mayores con la eliminación de los hortícolas que con los cítricos. Los efectos finales sobre el empleo son perceptibles para el caso de los cultivos hortícolas. En cuanto a los efectos indirectos sobre el sector industrial éstos son muy pequeños (alrededor del 1\%), mientras que para la economía en general, los efectos llegan al $2 \%$ debido en buena medida al efecto del multiplicador considerado $(1,4)$. Una primera aproximación permite ver el mayor impacto socioeconómico que supone la eliminación de los hortícolas frente a los cítricos, ya que representan una mayor superficie y demandan más mano de obra. Es importante tener en cuenta el papel amortiguador que puedan tener cultivos alternativos menos contaminantes y con presencia en la ZR, como pueda ser el olivar.

En el Cuadro 5 se presentan los costes directos e indirectos para cada medida, el CAE, medido en millones de euros por $\mathrm{m}^{3}$ año, así como el ACE que implica la puesta en marcha de cada medida de forma acumulada. El significado económico que se otorga en este trabajo al índice ACE equivale al concepto de coste marginal, pues representa el incremento de costes anuales que supone la puesta en marcha de cada medida por cada $\mathrm{mg} \mathrm{NO}_{3} / 1$ reducido en el acuífero. Este análisis se complementa con el Gráfico 7, en el que se presentan los costes totales asumidos con la implementación sucesiva de las medidas por cada punto de brecha reducida. De estos resultados se deduce el interés que tienen por su bajo coste marginal la medida de fertirrigación y la que conlleva la eliminación de los cítricos. Estos resultados obvian aspectos importantes como los costes hundidos asociados a la inversión en la depuradora ya que éstos difícilmente se van a recuperar en el futuro. Por el contrario, los costes de las inversiones que conllevan el resto de las medidas, sí son recuperables por su propia naturaleza -el trasvase y la compra de derechos lo financian los regantes y los programas de fertirrigación- pueden ser incluidos en programas de subvenciones presupuestados y aprobados por la Comisión Europea. 


\section{CUADRO 5}

\section{Costes asociados a cada medida}

\begin{tabular}{|c|c|c|c|c|c|}
\hline \multicolumn{2}{|c|}{ Medida } & $\begin{array}{l}\text { Costes Directos } \\
\text { ( mill. de euros) }\end{array}$ & $\begin{array}{l}\text { Coste Indirecto } \\
\text { ( mill. de euros) }\end{array}$ & $\begin{array}{c}\text { CAE } \\
\text { (mill. de euros/año) }\end{array}$ & $\begin{array}{c}\text { ACE } \\
\text { (mill. de euros/mg NO} \\
-/ 1)\end{array}$ \\
\hline \multicolumn{2}{|c|}{ Aplicación Directiva Nitratos } & 0,00 & 0,00 & 0,00 & 0,00 \\
\hline \multicolumn{2}{|c|}{$\begin{array}{l}\text { Desaladora + trasvase } \\
+ \text { compra derechos }\end{array}$} & 62,00 & $\begin{array}{c}\text { Efectos } \\
\text { terceras partes }\end{array}$ & 30,9 & 0,62 \\
\hline \multicolumn{2}{|c|}{ Programa fertirrigación } & 4,30 & $-0,62$ & 0,31 & 0,01 \\
\hline \multirow{3}{*}{$\begin{array}{l}\text { Eliminación } \\
\text { cultivos }\end{array}$} & Cítricos & 2,82 & 48,10 & 2,82 & 0,08 \\
\hline & Hort. & 15,46 & 263,70 & 15,46 & 0,33 \\
\hline & Citr. + hort & 18,21 & 312,80 & 18,21 & 0,14 \\
\hline
\end{tabular}

Fuente: Elaboración propia.

Las conclusiones asociadas a los resultados extraídos para la eliminación de cultivos podrían ser extrapolables a todas las ZR de la provincia y a todas sus masas sobreexplotadas. No obstante habría que tener en cuenta que, a pesar de que un abandono de la actividad puede conducir en el largo plazo a la recuperación ambiental, éste no podría ser asumido de forma global por el gran impacto que supondrían en la provincia y en la región. En este caso habría que considerar el beneficio ambiental asociado a cada masa y el coste a escala local. Se trataría de este modo de territorializar el problema y tratarlo desde un enfoque de abajo a arriba, para seleccionar aquellas masas sobre la que es prioritario actuar.

\section{GRÁFICO 7}

\section{Relación coste total reducción brecha contaminación}

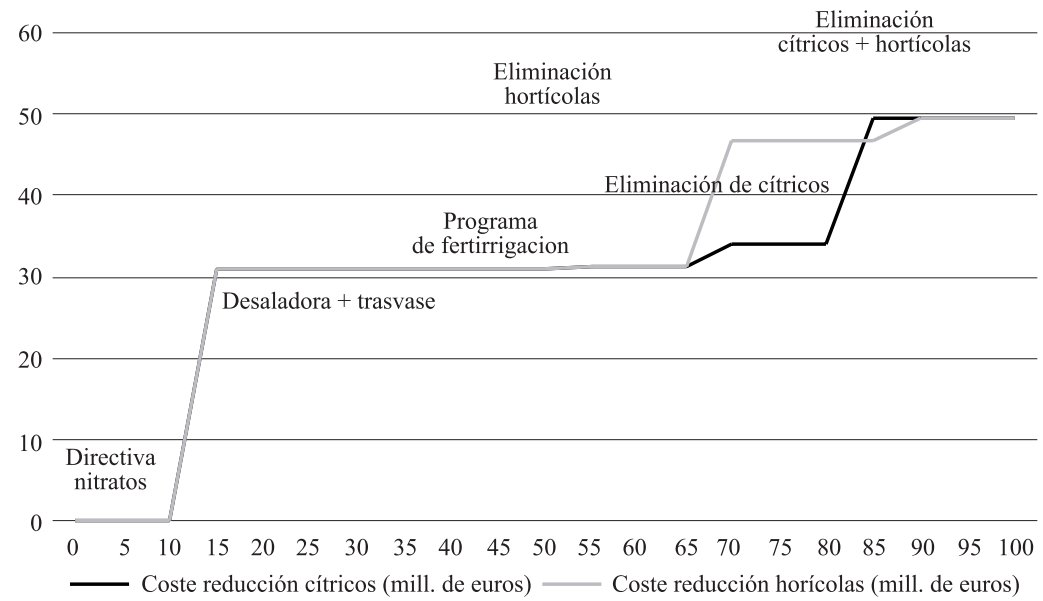




\section{Conclusiones}

Los principios de la DMA relativos a la inclusión de los objetivos ambientales en la gestión de los recursos hídricos, se están incorporando de forma paulatina al proceso de planificación hídrica que se lleva a cabo en la actualidad en las cuencas españolas. El ACE es el instrumento propuesto desde la UE para valorar el coste que conlleva la recuperación ambiental de las masas de agua, de forma que la información aportada debería incorporarse al proceso de decisión que conlleva dicha planificación. Sin embargo en el momento actual, las distintas demarcaciones no han terminado de incorporar el instrumento en el proceso de planificación, tomando decisiones en cuanto a la elección de las medidas a poner en marcha para mejorar el estado ambiental de las masas de agua siguiendo la inercia del pasado. En esencia, éstas se basan en la inversión en medidas correctoras que supongan una oferta adicional de recursos, sin valorar en ningún caso los problemas ligados a la calidad de las aguas. Este hecho es especialmente patente en las masas de agua subterráneas muy modificadas a causa de la presión que ejerce la actividad agraria, y que se localizan fundamentalmente en el sureste español.

Este trabajo se centra en este tipo de masas de aguas, tratando de aportar medidas concretas para ser incorporadas en el proceso de recuperación ambiental de estas masas de forma adicional a las inversiones en infraestructuras. Se trata en definitiva de aplicar los principios y metodología que la DMA propone, anteponiendo los objetivos de recuperación ambiental y abordando el cálculo de su coste. El acuífero aquí analizado presenta por el efecto de los lixiviados procedentes de la agricultura una contaminación por nitratos cinco veces superior a la admitida. La construcción de una desaladora y la incorporación al sistema de aguas trasvasadas desde la cuenca del Guadalquivir, solucionan de forma parcial los problemas ligados a la sobreexplotación del acuífero, en la medida que aportarán garantía de suministro al sistema en el momento que entren en funcionamiento. Sin embargo, en el Plan en proceso de aprobación no se incluye ningún tipo de medida que aborde de forma directa la corrección de los niveles de contaminación en el acuífero. Para alcanzar el objetivo de mejorar la calidad de las aguas es necesario plantear medidas directamente ligadas al uso del agua por el sector agrario.

Los resultados de este trabajo obtenidos a partir de la aplicación del ACE, muestran que las medidas básicas que simplemente supongan el cumplimiento de la DN o la aplicación de agua para riego libre de contaminantes tras la incorporación de fuentes alternativas de suministro, suponen una mejora importante en los niveles de contaminación y resolverían en buena medida los problemas de sobreexplotación de las masas de agua. Sin embargo, la recuperación de los niveles óptimos requiere la introducción de nuevas tecnologías ligadas al abonado localizado que muestran unas altas tasas de eficacia a un coste moderado. Ello es así porque el área de riego tiene una buena predisposición a estas tecnologías. Además, los resultados muestran el peso de los cítricos en el proceso de contaminación y la necesidad de actuar sobre ellos, bien a través de la mejora tecnológica o, incluso planteando su desaparición dada la menor incidencia en la economía de la zona respecto a otros cultivos alternativos como los 
hortícolas y el olivar. Es importante destacar que la metodología de estimación de la reducción de presiones en el acuífero requeriría la aplicación de métodos más precisos, que tengan en cuenta los factores edáficos, climáticos y geológicos que afectan a la dinámica de la incorporación de los lixiviados en el acuífero.

Se podría afirmar como consecuencia de este análisis, que es necesario controlar las extracciones reales del acuífero tras la incorporación de nuevas fuentes de suministro, así como, por supuesto, el control del cumplimiento de la Directiva de Nitratos, para que realmente puedan ser eficaces las medidas propuestas en lo que se refiere a la recuperación tanto cuantitativa como cualitativa del acuífero.

En cualquier caso, este trabajo se propone únicamente como un ejercicio de simulación de las distintas opciones de gestión del agua y del suelo, hasta ahora inabordables en estas zonas por la aceptación generalizada de la premisa del papel crucial del regadío en la economía de la región. Este ejercicio es más evidente en masas muy modificadas y de alto valor ambiental. Es por tanto preciso complementar el análisis con la valoración del beneficio social y ambiental que implica la recuperación del acuífero, así como los posibles efectos a nivel regional de una extrapolación de estos resultados al total de zonas regables que impacten sobre los acuíferos de las cuencas de la región. Se trataría en consecuencia de abordar el problema de la recuperación de las masas de un sistema desde un enfoque territorial, que tenga en cuenta los costes y los beneficios de la recuperación de cada masa y las implicaciones de ésta a escala local y regional. Esta información es fundamental y previa para la calificación final de los costes como desproporcionados, que implicaría la exención o aplazamiento del cumplimiento de los objetivos ambientales.

\section{Referencias}

ACUAMED. (2006). Informe de viabilidad de la desaladora en el Bajo Almanzora. Aguas de las Cuencas Mediterráneas S.A., Madrid.

Alcalá, F. y Sánchez, I. (2002). “Agua y producción agrícola: un análisis econométrico del caso de Murcia”. Estudios Agrosociales y Pesqueros, 197: 129-157.

Almasri, N.M. (2007). "Nitrate contamination of groundwater: A conceptual management framework”. Environmental Impact Assessment Review, 27(3): 220-242. http://doi.org/b48wr6

BPHCMA. (2011). Borrador del Plan Hidrológico de la Demarcación Cuencas mediterráneas Andaluzas. http://www.juntadeandalucia.es/medioambiente/portal_ web/agencia_andaluza_del_agua/gestion/gestion_agua_andalucia/planificacion. Consultado en diciembre de 2011.

Berbel, J., Mesa, P. y Martín-Ortega, J. (2009). “El análisis coste - eficacia de los programas de medidas". En Gómez-Limón, J.A. et al. (Eds.): La economía del agua de riego en España. Fundación Cajamar, Almería: 55-75. 
Cadahía, C. (2005). La fertirrigación. Cultivos hortícolas, frutales y ornamentales. Mundi-Prensa, Madrid.

Cardenete, M.A. y Sancho, F. (2003). "Evaluación de multiplicadores contables en el marco de una matriz de contabilidad social regional". Investigaciones Regionales, 2: 121-139.

Calatrava, J. y Gómez-Ramos, A. (2009). "El papel de los mercados de agua como instrumento de asignación de recursos hídricos en el regadío español”. En Gómez-Limón et al. (Eds.): La economía del agua en el regadio español. Fundación Cajamar, Almería: 295-319.

CIS. (2003). Common Implementation Strategy for the Water Framework Directive (2000/60/EC). Guidance Document No 1 Economics and the Environment - The Implementation Challenge of the Water Framework Directive. Office for Official Publications of the European Communities, Luxembourg.

Directiva 2000/60/CE del Parlamento Europeo y del Consejo, de 23 de octubre de 2000, por la que se establece un marco comunitario de actuación en el ámbito de la política de aguas.

Directiva 91/676/CEE del Consejo, de 12 de diciembre de 1991, relativa a la protección de las aguas contra la contaminación producida por nitratos utilizados en la agricultura.

Dworak, T. y Pielen. B. (2010). Selecting cost effective measures under the EU Water Framework Directive - The issue of scale. FEEM Working Paper, Milan.

Fezzi, C., Hutchins, M., Rigby, D., Bateman, I.J. Posen, P., Hadley, D. y Deflandre-Vlandas, A. (2010). "Integrated Assessment of Water Framework Directive. Nitrate Reduction Measures". Agricultural Economics, 41(2): 123-134. http://doi.org/dqd4rt

Garrido, A., Martínez-Santos, P. y Llamas, R. (2006). "Groundwater irrigation and its implications for water policy in semiarid countries: The Spanish experience". Hydrogeology Journal, 14(3): 340-349. http://doi.org/cfs9r4

Gil, M., Garrido, A. y Gómez-Ramos, A. (2010). "Economic analysis of drought risk. An application to irrigation agriculture in Spain". Agriculture Water Management, 98(5): 823-833. http://doi.org/cpw5nk

INE. (varios años). Contabilidad Regional de España. Instituto Nacional de Estadística, Madrid.

Junta de Andalucía. (2008). Inventario de Regadíos Andaluces. Consejería de Agricultura y Pesca, Sevilla.

Junta de Andalucía. (2006). Programa Regional de Desarrollo Rural de Andalucía 2007-2013. Consejería de Agricultura y Pesca, Sevilla.

Lacroix, A., Beaudoinb, N. y Makowski, D. (2005). “Agricultural water nonpoint pollution control under uncertainty and climate variability". Ecological Economics, 53(1): 115- 127. http://doi.org/cjr2w5 
Llamas, R. y Martínez-Santos, P. (2005). "Intensive groundwater use: silent revolution and potential source of social conflicts". Journal of Water Resources Planning and Management, 13(5): 337-341. http://doi.org/cvtbdm

Maestu, J. y Domingo, L. (2008). "Análisis coste-eficacia para la consecución de objetivos ambientales en masas de agua". Encuentros multidisciplinares, 10(29): 42-54.

Martínez, Y. y Albiac, J. (2004). "Agricultural pollution control under Spanish and European environmental policies". Water Resources. Research, 40: 1-12. http://doi.org/cwtxgn

Martín-Ortega, J., Berbel, J. y Brouwer, R. (2009). "Valoración económica de los beneficios ambientales de no mercado derivados de la mejora de la calidad del agua: una estimación de la aplicación de la Directiva Marco del Agua al Guadalquivir". Economía Agraria y Recursos Naturales, 9(1): 65-89.

MARM. (2009). Estudios de costes agrarios. Resultados Técnico-Económicos. Subdirección General de Análisis, Prospectiva y Coordinación, Madrid.

MARM. (varios años). Superficies comarcales por cultivos. Hojas 1T. Campaña 1995-2008. Ministerio de Medio Ambiente Rural y Marino, Madrid.

MARM. (varios años). Anuario de estadística agroalimentaria. Ministerio de Medio Ambiente Rural y Marino, Madrid.

MARM. (2008). Balance de Nitrógeno en la agricultura española. Año 2005. Ministerio de Medio Ambiente Rural y Marino, Madrid.

Mesa, P., Martín-Ortega, J. y Berbel, J. (2008). “Análisis multicriterio de preferencias sociales en gestión hídrica bajo la Directiva Marco del Agua". Economía Agraria y Recursos Naturales, 8(2): 105-126.

Olmedilla, S. y Gómez-Ramos, A. (2011). "Valoración coste eficacia de las transacciones de agua inter cuencas. Una aplicación las cuencas mediterráneas andaluzas". Observatorio Medio Ambiental, 14: 120-135. http://doi.org/bs5s9t

Polo, C., Roland-Host, D. y Sancho, F. (1991). "Descomposición de multiplicadores en un modelo multisectorial: una aplicación al caso español". Investigaciones Económicas, 15(1): 53-69.

Rodríguez, I. y Llanes, G. (2004). Matriz de contabilidad social y medioambiental: Aplicación a las emisiones de gases efecto invernadero de la Economía Española del año 2000. Estudios sobre economía aplicada. Serie EEE [CFB] $n^{\circ} 181$. Fedea, Madrid.

Sánchez-Picón, A., Aznar, J.A. y Aznar, P. (2011). "El trasvase Tajo-Segura en la provincia de Almería. Evolución e integración en la oferta de recursos hídricos del Valle del Almanzora”. En Melgarejo, J. (Ed.): El trasvase Tajo-Segura: repercusiones económicas, sociales y ambientales en la cuenca del Segura. Caja de Ahorros del Mediterráneo, Alicante.

Sauquillo, A. Custodio, E. y Llamas, R. (2009). "La gestión de las aguas subterráneas". Tecnología del Agua, 305: 60-67. 
Saz del, S., Hernández, F. y Sala, R. (2009). "Estimación del valor económico de la calidad del agua de un río mediante una doble aproximación: una aplicación de los principios económicos de la Directiva Marco del Agua”. Economía Agraria y Recursos Naturales, 9(1): 37-63.

Urbano, P. (1995). Tratado de fitotecnia general. Mundi-Prensa, Madrid.

Wustenberghs, H., Broekx, S., Van Hoof, K., Claeys, D., D’Heygere, T., D’Hooghe, J., Dessers, R., Huysmans, T., Lauwers, L., Meynaerts, E. y Vercaemst, P. (2008). "Cost-benefit analysis of abatement measures for nutrient emission from agriculture". Comunicación presentada al $12^{\text {th }}$ Congress of the European Association of Agricultural Economists - EAAE. Gent. 